\title{
El aprendizaje
}
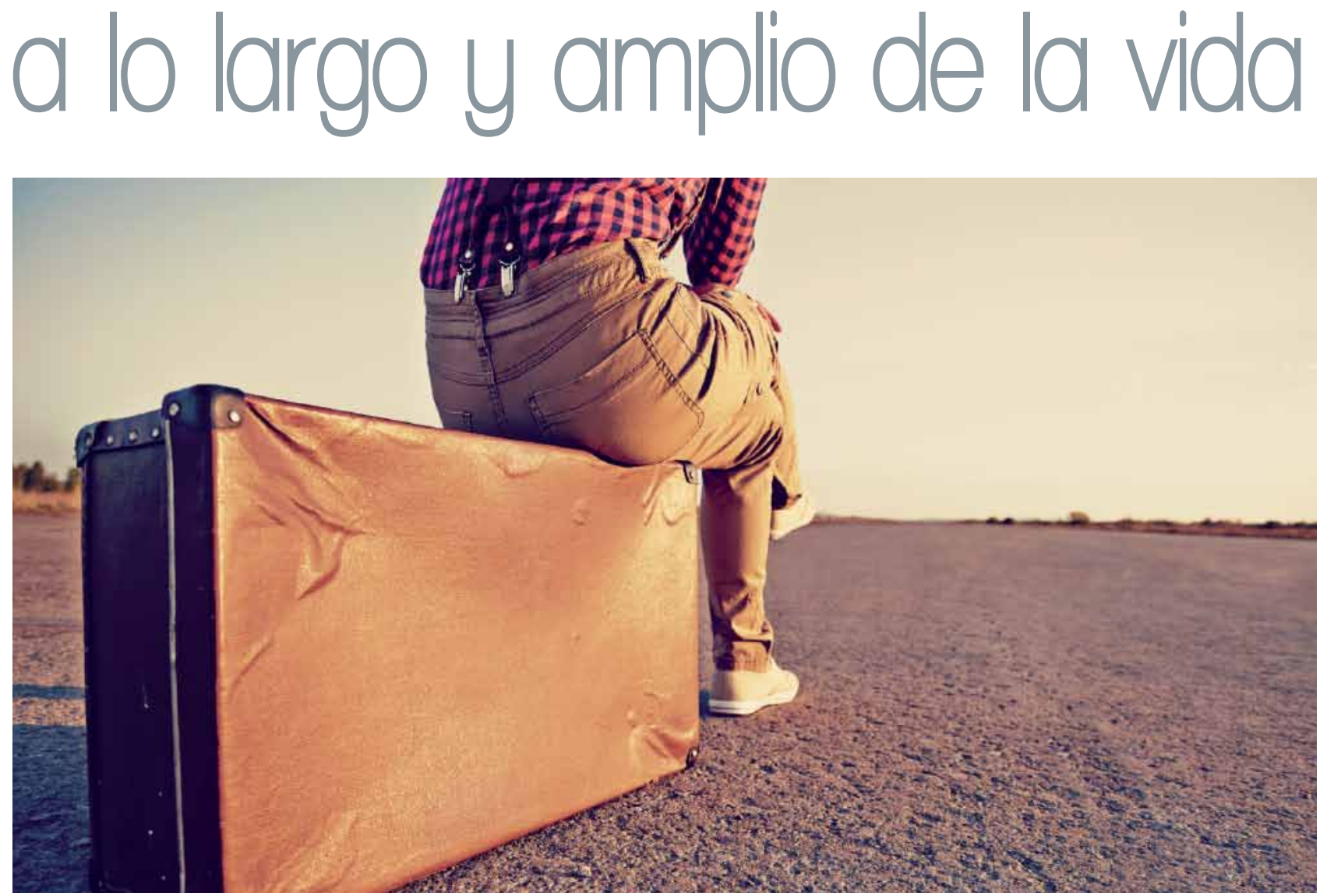

La nueva ecología del aprendizaje supone un cambio en el dónde y el cuándo aprender. La escuela sigue siendo un contexto imprescindible de aprendizaje, pero cada vez tienen más peso otros entornos informales.

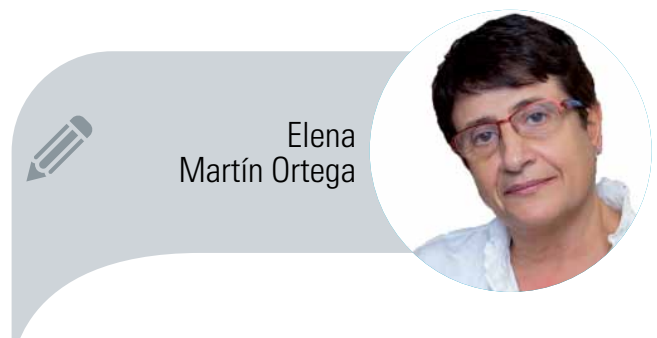
La necesidad de aprender trasciende los años de educación formal. Las distintas trayectorias de aprendizaje, que ahora están desconectadas, deberían integrarse y ser la base de una mayor personalización del aprendizaje. Esta nueva ecología conlleva cambios para la escuela y para la familia. 
Podemos dar por consensuado, en el discurso teórico, que la educación trasciende la escolarización; aprendemos y nos desarrollamos en distintos contextos, sólo uno de los cuales es la escuela. También se ha consolidado el acuerdo de que, por lo tanto, la coordinación de ésta con el resto de los entornos educativos es un elemento clave de la calidad de la enseñanza. Se asume asimismo la necesidad de que las instituciones escolares establezcan alianzas que trabajen con otros agentes sociales en una estructura de red que potencie las metas de todos ellos. La tesis que argumentaré en este artículo es que estos supuestos han implicado un cierto avance en el marco teórico que, sin embargo, es necesario articular en un enfoque global y que además en la mayoría de los casos no se ha traducido en nuevas prácticas educativas realmente innovadoras a pesar de que entrañan un cambio profundo en la manera de educar.

La afirmación que a nuestro juicio mejor sintetiza este enfoque global es que en la educación no sólo ha cambiado el qué y el cómo aprender; sino también y fundamentalmente el dónde se aprende y el cuándo se aprende (BANks, Au, Ball, Bell, Gordon, Gutiérrez, Heath, Lee, Lee, Mahiri, Nasir, Valdés y Zhouet, 2007). Las personas (y en especial los niños y jóvenes) aprenden muchas cosas fuera de la escuela y tendrán que seguir aprendiendo a lo largo de la vida, como consecuencia de la sociedad de la información (CASTELLS, 2000; Hung, Lim y JamaLudin, 2014; TEDEsco, 2000). Esto ha modificado lo que BARRON (2006) denomina "ecología del aprendizaje", es decir, el conjunto de contextos (físicos o virtuales) que ofrecen oportunidades de aprendizaje. Cada contexto implica una configuración propia de actividades, materiales, recursos, relaciones e interacciones que emergen de ellas.

Esta constatación tiene múltiples consecuencias, pero nos centraremos en las dos que consideramos más relevantes. Una primera derivada es el debate acerca de la posible pérdida de influencia de la escuela. ¿El hecho de que los alumnos y alumnas aprendan en otros entornos resta relevancia a los aprendizajes escolares? Sin duda implica cambiar profundamente la enseñanza, pero creemos que de hecho le atribuye mayor importancia, ya que es la institución responsable de conseguir que todos los estudiantes hayan adquirido los recursos necesarios para aprender en distintos contextos y para continuar haciéndolo cuando hayan acabado su educación formal. Enseñar a aprender a aprender, a lo "largo y amplio de la vida" (BAnks y OTROS, 2007), es pues la tarea específica e ineludible de la escuela. Aquellos centros que no preparen a su alumnado para este reto lo estarán colocando en una situación de desigualdad de oportunidades. La relevancia de la educación escolar estaría por tanto fuera de toda duda.

La otra consecuencia es el énfasis en la personalización del aprendizaje como eje vertebrador de la calidad de la enseñanza. Puede que al lector este término le resulte en principio poco estimulante, incluso manido. Sin embargo, supone un avance con respecto a la idea de atención a la diversidad que hasta ahora aunaba la aspiración de ofrecer una enseñanza de

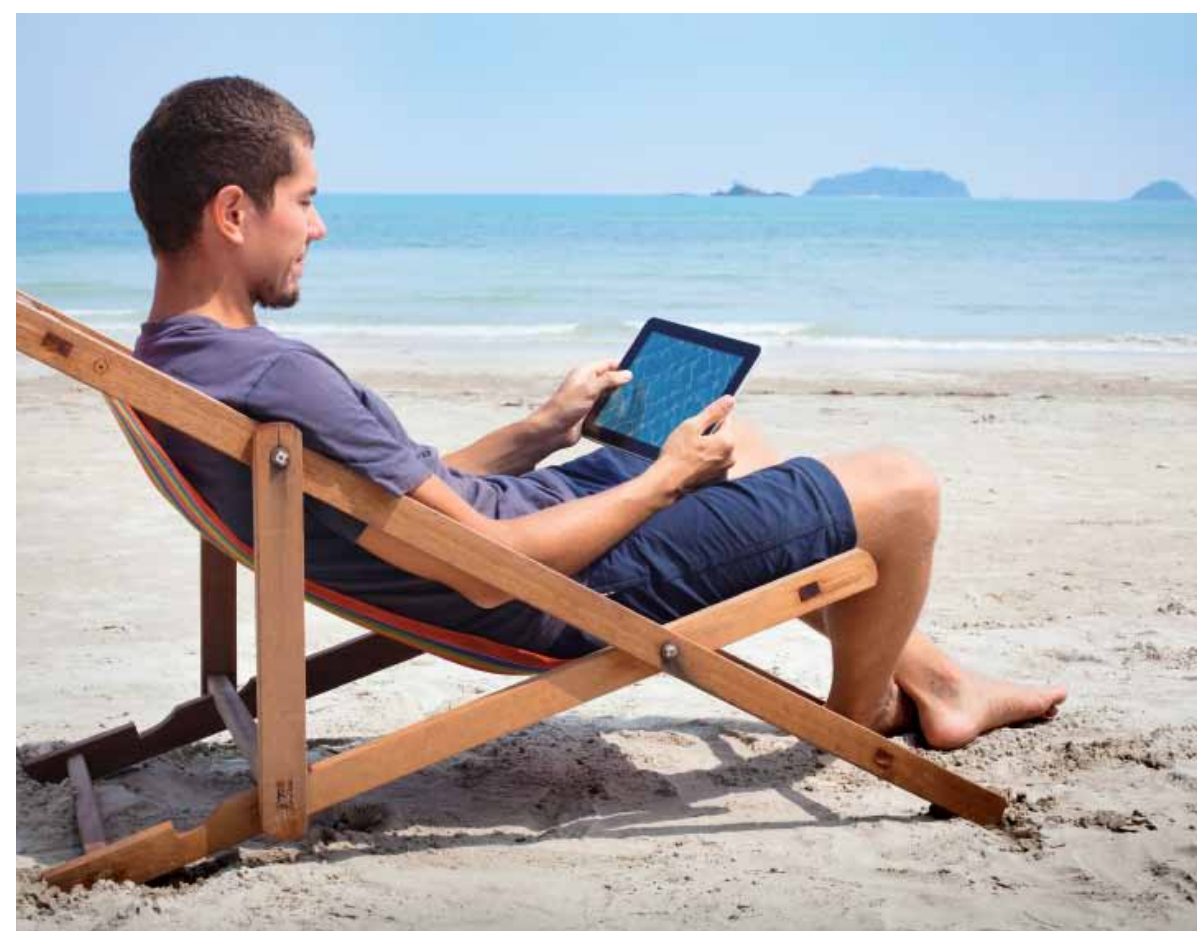




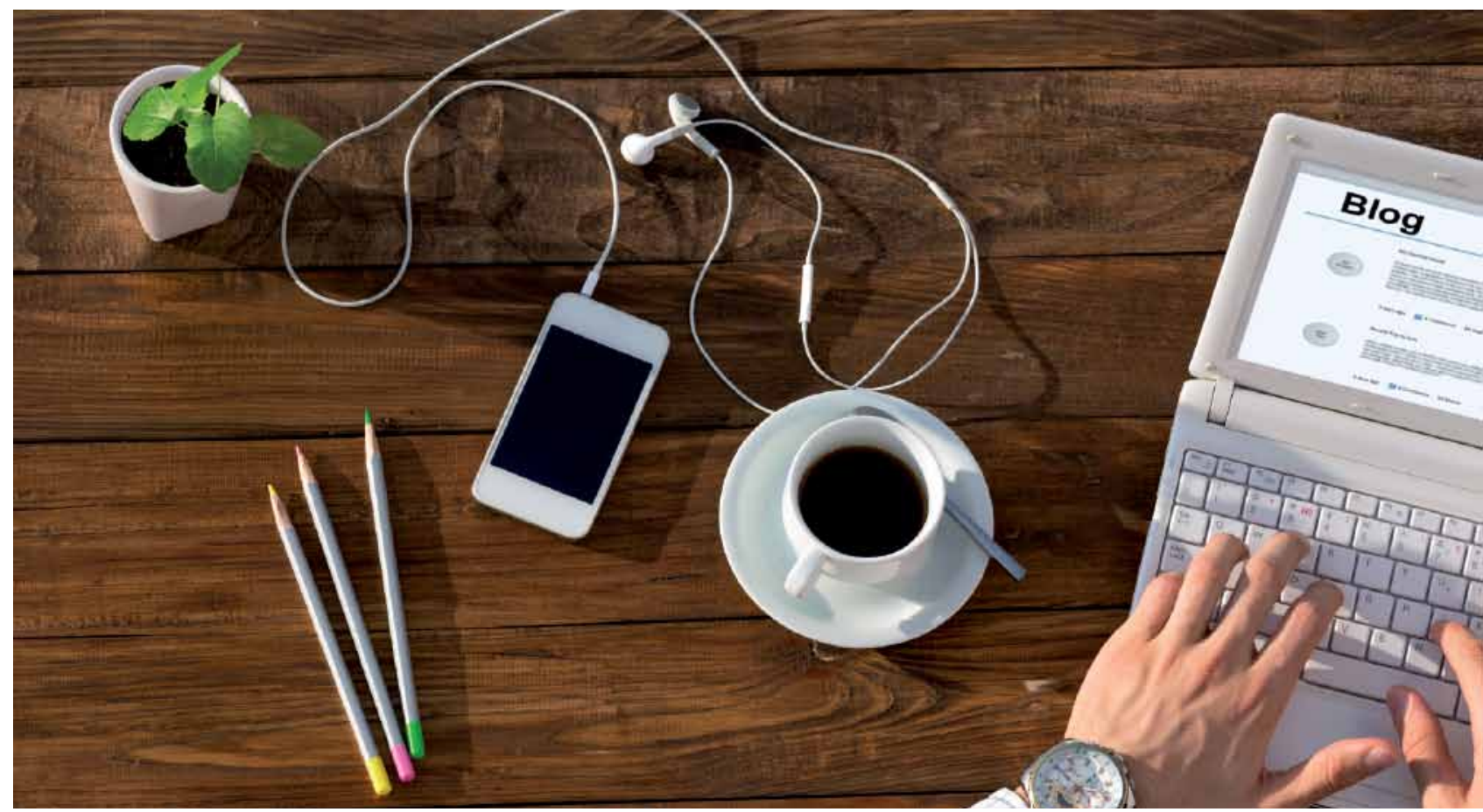

calidad a todos los alumnos en una escuela inclusiva. El concepto de diversidad sigue siendo clave, pero el de personalización añade el énfasis en las trayectorias individuales de aprendizaje. Niños y jóvenes se construyen como aprendices en distintos contextos educativos que es preciso ayudar a articular en una experiencia integradora en cuya configuración tiene un papel esencial el propio alumno. En palabras de César Coll:

[...] el alumno no es simplemente alguien con unas características que se han de tener en cuenta y con unas necesidades de aprendizaje que se han de satisfacer: es ante todo alguien con voz y capacidad reconocida para participar, a partir de sus características, aspiraciones e intereses, en la identificación de necesidades de aprendizaje y en la definición y control del camino para satisfacerlas (CoLl, en prensa, p.10.)

La creciente influencia de otros contextos educativos (prácticas sociales de ocio o escenarios informales como museos, ludotecas, entornos de aprendizaje o de juego on-line...), si bien no resta valor a los que vienen estando presentes (familia y escuela), requiere en ellos una profunda transformación que debe partir de supuestos comunes. ¿Cuáles serían los ejes clave de estos cambios?

\section{Líneas maestras en los centros escolares}

La escuela tiene que favorecer que los alumnos vayan tomando conciencia de su trayectoria de aprendizaje no sólo en relación con las experiencias que tienen lugar en los tiempos y espacios escolares; sino con todas aquéllas que forman parte de su vida cotidiana. La elaboración de planes personalizados de aprendizaje puede parecer una meta inalcanzable, pero no lo es si se toma la perspectiva temporal de toda la educación obligatoria y de un equipo docente conjunto que ejerce influencia educativa en coordinación con la familia; planes con un foco amplio y a largo plazo que confieren identidad propia y compromiso compartido a la escolaridad completa de cada alumno. Personalizar el aprendizaje no significa hacer cosas distintas con cada alumno. Significa planificar las actividades de manera que puedan realizarse de formas distintas (ritmos y estilos de aprendizaje diversos, énfasis diferentes, intereses y motivaciones variadas, etcétera).

El aprendizaje tiene su origen en las actividades y experiencias de los niños y jóvenes; en el caso de la escuela la actividad no es suficiente ya que el objetivo no es sólo actuar sobre el mundo sino comprenderlo. Pero esta función epistémica no debe hacernos olvidar que la clave del aprendizaje está en el sentido que las 
actividades tienen para los aprendices. El sentido no es una condición previa, no significa que haya que plegarse tan sólo a los intereses que espontáneamente muestran los niños y jóvenes. El sentido se construye, pero nunca al margen de la actividad. De sus características depende en gran medida el nivel de motivación y de compromiso del alumno.

La relevancia de las actividades va unida a su vez a su autenticidad. En el contexto escolar esta demanda se traduce en la selección de los aprendizajes pero sobre todo en las metodologías utilizadas. Las metodologías basadas en proyectos, problemas o talleres ofrecen experiencias con mayor sentido (MARCHESI y MARTín, 2014). La artificialidad de la organización escolar en una secuencia de materias que deben interrumpirse cada hora no puede seguir manteniéndose. Los tiempos y los espacios deben estar al servicio de una organización que promueva actividades con sentido dentro de los planes personalizados.

Pero un plan personalizado no se limita a las experiencias escolares; sino que integra aquellas otras que son clave en la vida del alumno o alumna. La identidad de aprendiz de muchos de ellos puede que pivote sobre las habilidades sociales que desarrolla con su grupo de amigos, el deporte que ejercita, la música que consume o produce, el voluntariado que realiza, las actividades literarias que Ileva a cabo (teatro, cuentacuentos, blogs...). Los docentes atentos y sensibles siempre han sabido identificar el valor de estas experiencias de aprendizaje para el desarrollo de sus alumnos y, más en concreto, para el éxito escolar. Todo niño o joven tiene ámbitos en los que brilla. El riesgo está en que la escuela desperdicie estos anclajes del sentido del aprendizaje porque no se conocen o porque no se consideran valiosos.

Esta vía de avance supone también construir lazos estables con otros contextos educativos. Una vez más, el punto de inflexión estaría en trascender el carácter anecdótico de esta vinculación. No nos referimos a hacer una salida a un museo o a un evento musical, sino a contar de

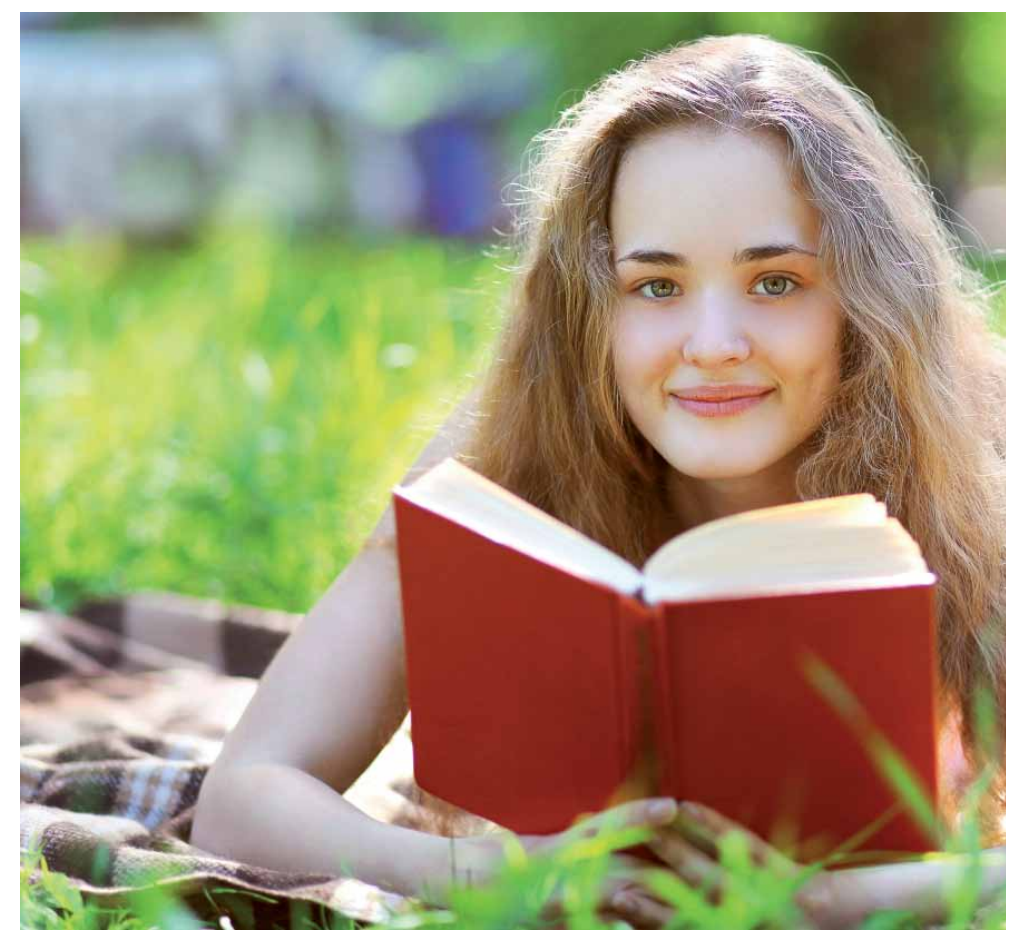

forma continuada y sustantiva con estos recursos cuando hacemos la programación; a superar la tendencia endogámica que caracteriza a la escuela y asumir en la práctica que nos entrelazamos sin por ello diluirnos. El enfoque del "aprendizaje conectado" es una clara línea de futuro.

¿Es viable una propuesta de este tipo? Sin duda implica más trabajo, pero el balance resulta a todas luces positivo. La mayor desazón de los docentes viene de la perdida de sentido que muestra en muchos casos el alumnado. El desenganche de una parte importante de los alumnos es una realidad constatable, pero que puede modificarse, ya que no sé debe a un cambio en la esencia de las nuevas generaciones sino en las prácticas sociales y en las nuevas demandas que implica para la escuela la sociedad de la información. Como toda innovación, impulsarla exige diseñar procedimientos compartidos por el conjunto del equipo docente y protocolizarlos hasta que se conviertan en una rutina; en una seña de identidad de la cultura del centro.

\section{Implicaciones para las familias}

Reconceptualizar la educación desde esta perspectiva, es decir, entender que el aprendizaje se lleva a cabo en distintos contextos educativos que contribuyen todos ellos de forma muy relevante al desarrollo de niños y jóvenes, conlleva 


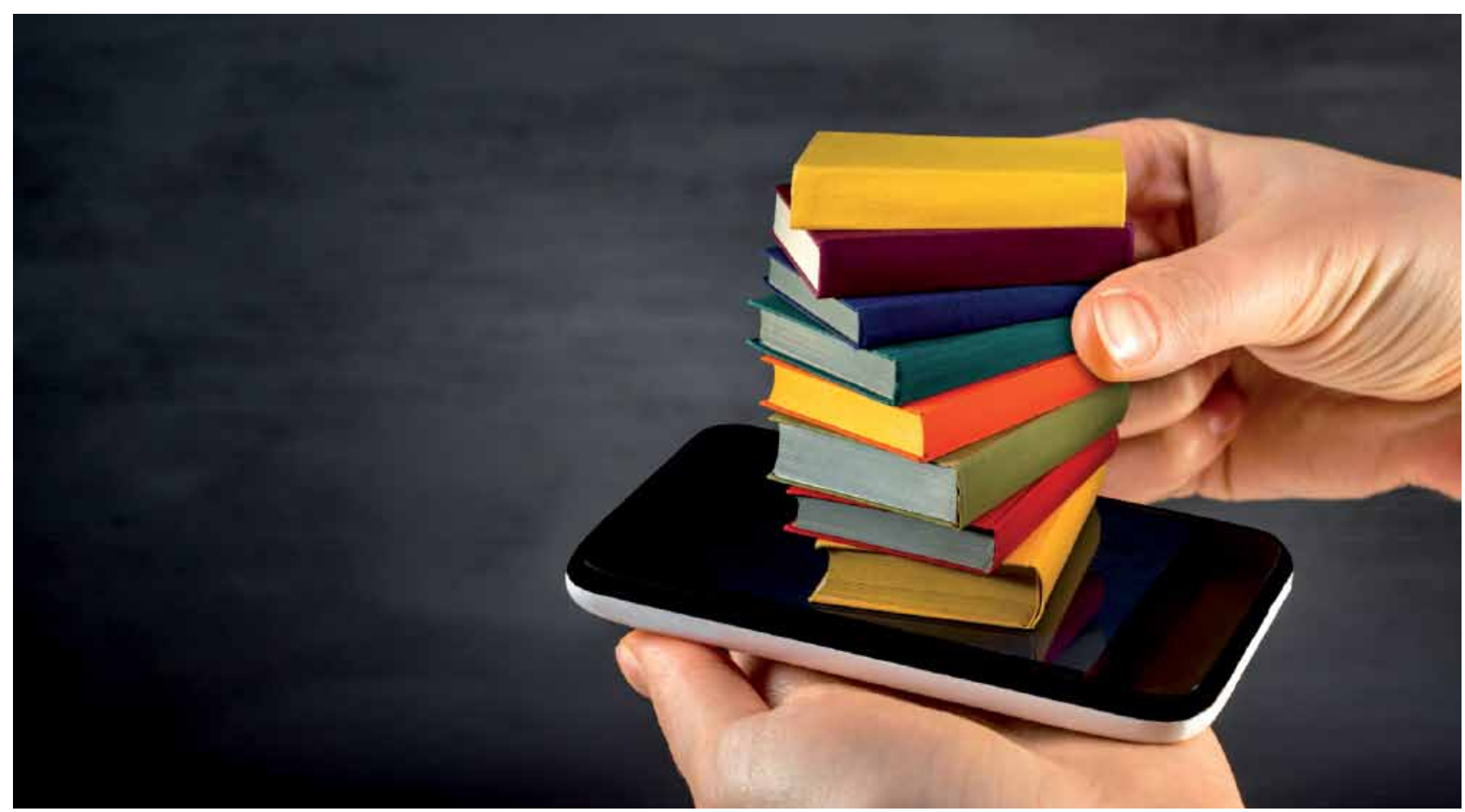

también cambios notables en las funciones de los progenitores ${ }^{1}$. Uno de ellos se refiere a la coordinación con la escuela. ¿Quién mejor que la familia puede ayudar a los docentes a conocer esa parte de la vida del alumno que les es ajena? No se trata de utilizar a los padres como meros informantes sino de compartir el diseño de las distintas trayectorias de aprendizaje, desde un estatus de auténtica corresponsabilidad. En una relación equilibrada, los progenitores no intentarán invadir el espacio de competencia de los docentes respetando su pericia en el ámbito instruccional; el profesorado, por su parte, respetará y valorará los espacios de decisión de los progenitores y reconocerá sus recursos sin adoptar posiciones de superioridad. Orientar a las familias cabe en esta relación, pero no desde una concepción que considera que éstas no están haciendo bien sus funciones y es necesario definírselas desde las necesidades de la escuela. Los planes personalizados de aprendizaje deberían por tanto elaborarse conjuntamente y servir de guía para ambas instituciones educativas.

1 Utilizamos este término para referirnos a los padres y madres y a cualquier otra figura que ejerza las responsabilidades que la parentalidad implica.
Pero las funciones de la familia trascienden la coordinación con la escuela precisamente porque ella sí está presente en los distintos contextos educativos. Como señala Rodrigo y Palacios (1998), los progenitores ejercen una influencia educativa directa sobre el sujeto en desarrollo, pero también una indirecta a través de la estructuración del resto de contextos educativos en el que éste se desenvuelve. Son los progenitores quienes eligen el colegio de sus hijos, quienes organizan sus rutinas de ocio y con ello en gran medida sus relaciones sociales; quienes, en último término, configuran las prácticas sociales en las que van a participar y aprender.

Desde esta perspectiva, la elección del colegio o instituto es una decisión de mucha responsabilidad que no siempre está suficientemente fundamentada. ¿Qué criterios usan los progenitores para esta selección? ¿Responden a opciones fundamentales de modelo educativo o relacionadas más bien con el tipo de población escolar o los recursos materiales del centro? ¿Ofrecen los centros información suficiente para que las familias puedan valorar las señas de identidad de los distintos proyectos? Para apoyar este proceso sería necesario, por una parte, que los centros lo organizaran desde el convencimiento de que las familias tienen que comprometerse con la escolaridad de sus hijos y que la meta trasciende por tanto el objetivo más ligado al 


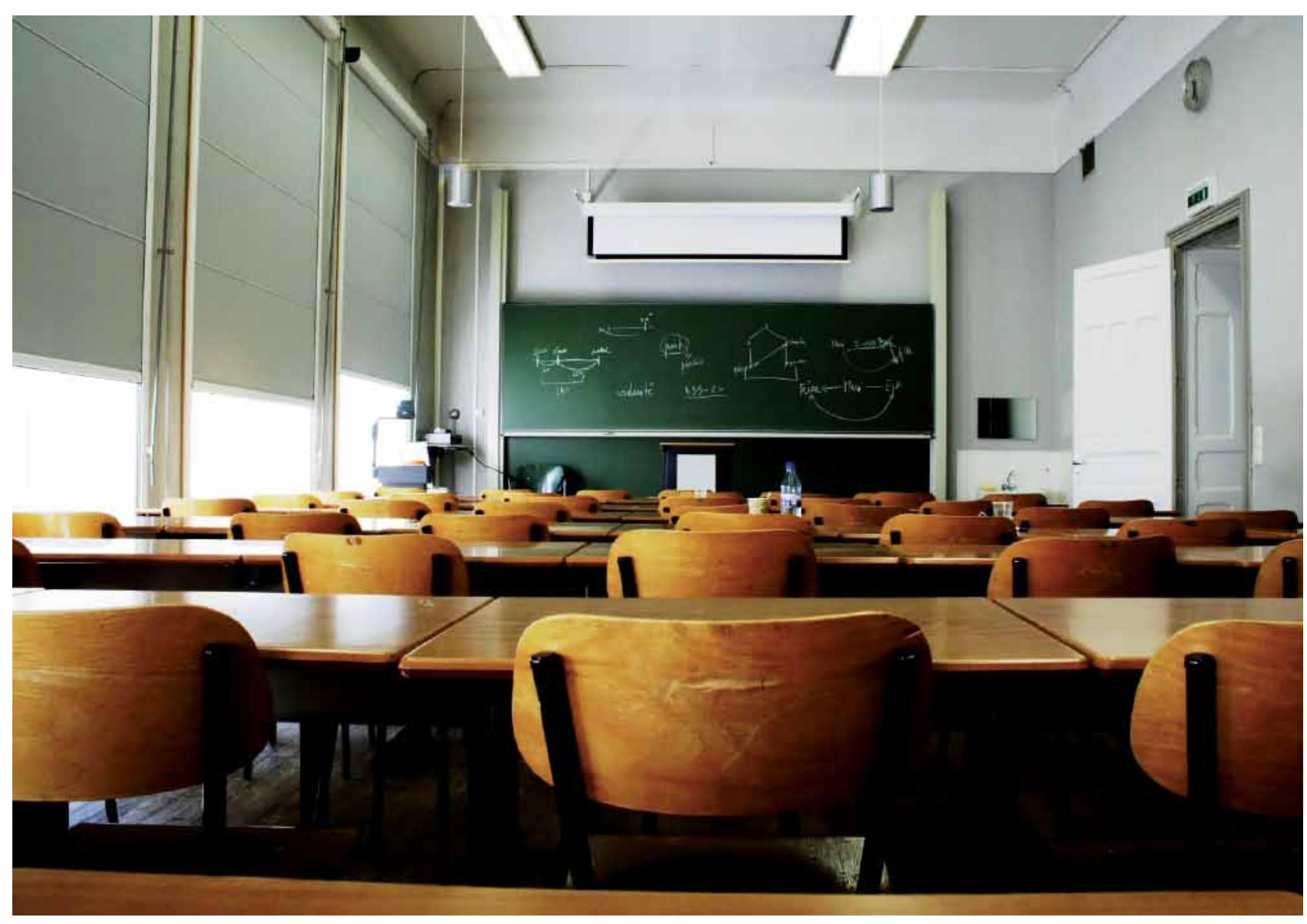

marketing. Por otra, sería bueno reconocer que no se trata una decisión sencilla y que habría que formar a los progenitores para que comprendieran la complejidad que conlleva, las consecuencias que se derivan y la colaboración que va a requerir por su parte.

¿Cuánto tiempo pueden dedicar los hijos e hijas a las actividades extraescolares? ¿Cuáles son las más adecuadas? ¿Qué peso debe tener su opinión en la decisión? Los criterios para estructurar el ocio de niños y jóvenes tampoco son fáciles de establecer y no está garantizado que los progenitores vayan a estar acompañados por expertos cuando tienen que tomar decisiones en este ámbito. Sin pretender dar recetas simplistas, existe conocimiento fundamentado que permite orientar a las familias. Las actividades extraescolares son positivas siempre que aseguren un tiempo diario para el juego libre y para las tareas escolares (MARSH y KLEITMAN, 2002). No hay un horario predefinido, el equilibrio entre estos tres elementos imprescindibles para el desarrollo es la clave del éxito. Es preciso ajustar la organización a cada
¿Dónde encuentran las familias ayuda

para lomar con acierto las numerosas y

relevantes decisiones que acaban configurando

los diversos contextos educativos

de sus hijos e hijas?

caso y revisarla periódicamente, sin vivir como un fracaso que pueda abandonarse una actividad. Estas actividades deben ser experiencias que resulten satisfactorias para los niños y jóvenes, lo que no significa que no impliquen esfuerzo y tenacidad. Y no deben entenderse única ni principalmente como refuerzo de habilidades académicas.

Por último, ¿qué parámetros deben tener en cuenta los progenitores para regular el uso de las TIC? Un reciente estudio de la Unión Europea, realizado con familias con hijos entre 0 y 8 años (RC, 2015; Matsumoto, Aliagas, Morgade, Correro, Galera, RonceRo y Poveda, 2016), pone de manifiesto que 


\section{(D.) DARA SABER MÂS}

Banks, J., Au, K., Ball, A., Bell, Ph., Gordon, E., Gutiérrez, K., Heath, Sh., Lee, C., Lee, Y., Mahiri, J., Nasir, N., Valdés, G., y Zhouet, M. (2007). Learning In and Out of School in Diverse Environments:LifeLong, Life-Wide, Life-Deep. The Learning in Informal and Formal Environments Center. University of Washington, Stanford University, and SRI International. Consultado el 20.1.2016 en http:// lifes/c.org/docs/Banks_etal-LIFE-Diversity-Report.pdf

BARRON, B. (2006). Interest and self-sustained learning as catalysts of development: A learning ecologies perspective. Human Development, 49, 193-224.

Castells, M. (2000). La era de la información, Vol.1. La sociedad red. Madrid: Alianza.

Coll, C. (2013b). El currículo escolar en el marco de la nueva ecología del apredizaje. Aula de Innovación Educativa, 219, 31-36.

Coll, C. (en prensa). La personalització de l'aprenentatge escolar: un repte indefinible. En J. M. Vilalta (Coord.), Anuari de l'Educació a Catalunya 2016. Barcelona: Editorial Mediterrània.

Hung, K. Y. T. Lim K.Y.T. \& Jamaludin (2014). An Epistemic Shift: A Literacy of Adaptivity as Critical for Twenty-First Century Learning. En D. Hung, K.Y.T. Lim \& Sh-Sh. Lee (pp. 3-14). Adaptivity as a Transformative Disposition for Learning in the 21st Century. Singapore: Springer.

ito, M., Gutiérrez, K., Livingstone, S., Penuel, B. Rhodes, J., Salen, K., Schor, J., Sefton-Green, J. \& Watkins, S.C. (2013). Connected Learning: An Agenda for Research and Design. Irvine, CA: Digital Media and Learning Research Hub. Consultado 27.06.15 en http://dmlhub.net/wp-content/uploads/files/Connected_Learning_report.pdf

JRC (2015). Young children and digital technology: A qualitative exploratory study; $2015-2^{\text {nd }}$. Consultado el 22 de marzo de 2016 en http:// publications.jrc.ec.europa.eu/repository/handle/JRC93239

Kumpulainen, K. (2014). Pedagogies of Connected Learning: Adapting Education into the Twenty-First Century. En: Hung, D., LIM, K. Y.T. \& LeE, SH-SH. (Eds.), Adaptivity as a Transformative Disposition for Learning in the 21st Century (pp. 31-41). Singapore: Springer.

MARChesi, A. y Martín, E. (2014). Calidad de la enseñanza en tiempos de crisis. Madrid: Alianza.

Marsh, H. y Kleitman, S. (2002). Extracurricular school activities: the good, the bad, and the nonliear. Harvard Educational Review, 72, 4, pp. 464-514.

Matsumoto, M., Aliagas, C., Morgade, M., Correro, C., Galera, N., RonCERo, C. \& PovedA, D. (2016). Young children and digital technology: A qualitative exploratory study. National report: Spain. Consultado el 22.3.2016 en https://repositorio.uam.es/handle/10486/669336

Rodrigo, M.J. y Palacios, J. (1998). Familia y desarrollo humano. Madrid: Alianza.

Tedesco, J. C. (2000). Educar en la Sociedad del conocimiento. Buenos Aires: Fondo de Cultura Económica. el tiempo dedicado a las tecnologías digitales no está poniendo en peligro el resto de las actividades de ocio; que los progenitores intentan ejercer control tanto sobre el tiempo como sobre los contenidos a los que acceden sus hijos sin tener suficientemente en cuenta que hay que promover una progresiva autorregulación; y que los progenitores no siempre tienen claros los riesgos ni aprovechan todas los beneficios de las TIC.

¿Dónde encuentran las familias ayuda para tomar con acierto las numerosas y relevantes decisiones que acaban configurando los diversos contextos educativos de sus hijos e hijas? Resulta realmente ingenuo y peligroso creer que las familias están bien preparadas para cumplir con las máximas garantías con esta función de estructuración. La existencia de recursos de apoyo familiar, en las distintas formas que estos pueden tomar, son una de las manifestaciones más claras de que la sociedad ha entendido que la educación es más que la escolarización, y constituyen una vía prometedora para avanzar en coherencia con la nueva ecología del aprendizaje. La escuela tiene una responsabilidad ineludible en esta tarea, pero yo no es la única ni necesariamente la instancia más importante en ella.

Ojala estas breves reflexiones sirvan como homenaje a todos los padres y maestros (y madres y maestras) y contribuyan a celebrar como se merece los 50 años de la revista.

\section{HEMOS HABLAOO DE}

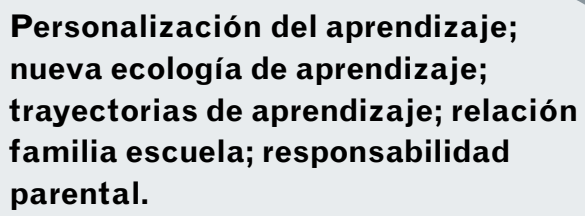

Este artículo fue solicitado por PADRES y MAESTROS en septiembre de 2015, revisado y aceptado en enero de 2016. 